University of Wollongong

Research Online

Faculty of Engineering and Information

Faculty of Engineering and Information

Sciences - Papers: Part A

Sciences

$1-1-2016$

\title{
High intrinsic sensitivity etched polymer fiber Bragg grating pair for simultaneous strain and temperature measurements
}

\author{
Kishore Bhowmik \\ University of New South Wales, National ICT Australia, NICTA \\ Gang-Ding Peng \\ University of New South Wales \\ Yanhua Luo \\ University of New South Wales \\ Eliathamby Ambikairajah \\ University of New South Wales, National ICT Australia, NICTA \\ Vedran Lovric \\ University of New South Wales
}

See next page for additional authors

Follow this and additional works at: https://ro.uow.edu.au/eispapers

Part of the Engineering Commons, and the Science and Technology Studies Commons

\footnotetext{
Research Online is the open access institutional repository for the University of Wollongong. For further information
} contact the UOW Library: research-pubs@uow.edu.au 


\title{
High intrinsic sensitivity etched polymer fiber Bragg grating pair for simultaneous strain and temperature measurements
}

\begin{abstract}
A sensing configuration for simultaneous measurement of strain and temperature with enhanced intrinsic sensitivity based on a fiber Bragg grating (FBG) pair with one grating inscribed in the etched and the other in unetched polymer fiber region is demonstrated. A poly (methyl methacrylate) based single-mode polymer fiber is etched to different diameters, and it is observed that etching can lead to change in the material properties of the fiber, such as Young's modulus and thermal expansion coefficient, which can play a vital role in improving its intrinsic sensing capabilities. Thus, exploiting the different strain and temperature sensitivities exhibited by etched and unetched polymer FBGs, strain and temperature can be simultaneously measured with very high sensitivity. Experimental results show that rms deviations of $\pm 8.42 \mu \in$ and $\pm 0.39^{\circ} \mathrm{C}$ for strain and temperature, respectively, in a real simultaneous measurement. The effect of individual thermal and strain sensitivity coefficients on measurement accuracy is also analyzed.
\end{abstract}

\section{Keywords}

grating, bragg, fiber, polymer, measurements, etched, temperature, sensitivity, intrinsic, high, strain, simultaneous, pair

\section{Disciplines}

Engineering | Science and Technology Studies

\section{Publication Details}

K. Bhowmik, G. Peng, Y. Luo, E. Ambikairajah, V. Lovric, W. R. Walsh \& G. Rajan, "High intrinsic sensitivity etched polymer fiber Bragg grating pair for simultaneous strain and temperature measurements," IEEE Sensors Journal, vol. 16, (8) pp. 2453-2459, 2016.

\section{Authors}

Kishore Bhowmik, Gang-Ding Peng, Yanhua Luo, Eliathamby Ambikairajah, Vedran Lovric, William R. Walsh, and Ginu Rajan 


\title{
High Intrinsic Sensitivity Etched Polymer Fibre Bragg Grating Pair for Simultaneous Strain and Temperature Measurements
}

\author{
Kishore Bhowmik, Student Member, IEEE, Gang-Ding Peng, Yanhua Luo, Eliathamby Ambikairajah, Vedran \\ Lovric, William R. Walsh and Ginu Rajan
}

\begin{abstract}
A sensing configuration for simultaneous measurement of strain and temperature with enhanced intrinsic sensitivity based on a fibre Bragg grating pair (FBG) with one grating inscribed in the etched and the other in un-etched polymer fibre region is demonstrated. A PMMA based singlemode polymer fibre is etched to different diameters and it is observed that etching can lead to change in the material properties of the fibre such as Young's modulus and thermal expansion coefficient, which can play a vital role in improving its intrinsic sensing capabilities. Thus, exploiting the different strain and temperature sensitivities exhibited by etched and un-etched polymer FBGs, strain and temperature can be simultaneously measured with very high sensitivity. Experimental results show that $\mathrm{rms}$ deviations of $\pm 8.42 \mu \epsilon$ and $\pm 0.39{ }^{0} \mathrm{C}$ for strain and temperature respectively in a real simultaneous measurement. The effect of individual thermal and strain sensitivity coefficients on measurement accuracy is also analysed.
\end{abstract}

Index Terms-Polymer optical fibre (POF), fibre Bragg grating (FBG), etching, strain, temperature.

\section{INTRODUCTION}

$\mathbf{F}$ IBRE Bragg grating (FBG) has been emerged as a promising sensing element owing to its potential for the measurement of several important physical parameters such as strain, temperature and pressure. Sensors based on polymer optical fibres (POFs) are many times more sensitive than silica fibre gratings because of their unique properties such as low Young's modulus, large strain measurement range, high temperature sensitivity and biocompatibility [1]. Since the first demonstration of a polymer optical fibre Bragg grating (POFBG) in 1999 [2], it has been used in different application areas [3], [4]. More recently the interest in POFs has increased and different applications of POFBG sensors such as for strain, temperature, humidity and pressure measurements have been reported [5], [6]. But for this measurements, one of technical issue that needs to be addressed is the inability

K. Bhowmik and E. Ambikairajah are with the School of Electrical Engineering and Telecommunications, UNSW, Australia and also with the ATP Research Laboratory, National ICT Australia (NICTA) (e-mail: k.bhowmik@student.unsw.edu.au).

G. D. Peng and Y. Luo are with the School of Electrical Engineering and Telecommunications, UNSW, Australia.

V. Lovric and W. R. Walsh are with Surgical \& Orthopaedic Research Laboratories, Prince of Wales Clinical School, UNSW, Australia.

G. Rajan is with the School of Electrical, Computer Telecommunications Engineering, University of Wollongong, Australia and also with the School of Electrical Engineering and Telecommunications, UNSW, Australia (e-mail: ginu@uow.edu.au). of a FBG sensor to eliminate its cross sensitivity effect. When FBGs are applied to the measurement of strain then their cross-sensitivity to temperature is an issue that needs to be addressed. One approach for solving this is to design sensing heads that are insensitive to temperature. Another is to develop schemes with sufficient degrees of freedom to permit simultaneous differentiation of these two parameters. Many researchers have worked on this problem and proposed a number of configurations.

The first sensing head reported for strain and temperature measurements was proposed and demonstrated by $\mathrm{Xu}$ et al [7] where two superimposed FBGs at two different Bragg wavelengths are used and the result shows $\pm 5{ }^{0} \mathrm{C}$ and $\pm 10 \mu \epsilon$ error for temperature and strain respectively. This concept of sensitivity differentiation has been widely explored afterwards and used in different configurations for simultaneous measurements of strain and temperature. James et al [8] proposed splicing two fibres of different diameters where the gratings showed similar temperature sensitivities but different strain responses. Liu et al [9] reported that, a combination of a POFBG and silica FBG was suitable for simultaneous measurements and provided large discrimination against temperature and strain. A number of similar techniques for simultaneously measuring temperature and strain has been proposed, including the use of two types of FBGs such as type IA/IIA [10] and type I/IIA [11], a FBG written on germanosilicate and boron-codoped germanosilicate fibres [12], the combination of a FBG and a long period grating [13], the combination of a FBG and an EDFA [14], two FBGs with different cladding diameters [15], a Mach-Zehnder discriminator and spliced different diameter fibre gratings [16], a highspatial resolution simultaneous measurement in time domain using a Panda fibre [17] and two FBGs embedded in a glass tube [18]. Although these methods may distinguish the crosssensitivity effect between strain and temperature, but most of them rely on using two different types of fibres and FBGs or any extrinsic special technique. As the use of more than one fibre element increases the system cost and complicates fabrication of the sensor head, it is highly desirable to utilise a single fibre without any extrinsic technique to achieve discrimination between strain and temperature. So any technique utilizing the same fibre without using any extrinsic techniques would be valued higher and also cost effective for simultaneous measurements. Moreover, for certain applications such as in biomedical/biomechanical sensors, higher sensitivity is 
TABLE I

MEASURED YOUNG'S MODULUS OF THE POLYMER FIBRE

\begin{tabular}{|c|c||c|c|}
\hline \multicolumn{2}{|c|}{ Sample-1 } & \multicolumn{2}{c|}{ Sample-2 } \\
\hline \hline Fibre Diameter $(\mu \mathbf{m})$ & Young's Modulus $(\mathbf{G P a})$ & Fibre Diameter $(\mu \mathbf{m})$ & Young's Modulus $(\mathbf{G P a})$ \\
\hline 180 (un-etched) & 2.56 & 450 (un-etched) & 1.04 \\
\hline 100 (etched) & 2.07 & 324 (un-etched) & 1.05 \\
\hline 70 (etched) & 1.71 & 340 (etched) & 0.36 \\
\hline
\end{tabular}

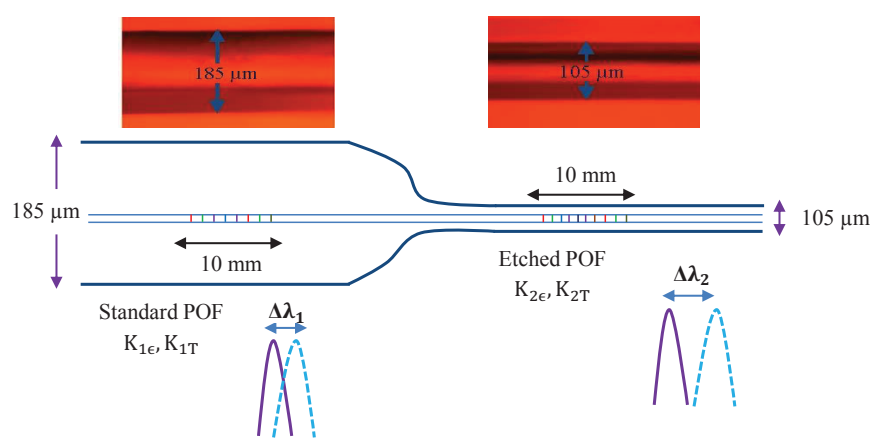

Fig. 1. Schematic diagram of fabricated grating pair for simultaneous measurement (inset: Microscopic images of the POF before and after etching)

required, so it's most important to ensure the high sensitivity at the same time. Therefore, any technique for simultaneously measuring temperature and strain and intrinsically improving sensitivity will be more highly valued than other existing techniques.

In this paper, we proposed a technique where a combination of a pair of etched and un-etched polymer FBG functions as a sensor system that simultaneously measures strain and temperature. In addition, utilising the material property changes exhibited in the polymer fibre due to etching, both temperature and strain sensitivity can be intrinsically enhanced.

\section{SENSOR DESIGN AND PRINCIPLE}

The photosensitive polymer fibre used in this experiment was an in-house produced step-index single-mode fibre, one of the highest photosensitive fibre reported so far [19]. The single-mode POF sample used had an outer diameter of $\sim 185$ $\mu \mathrm{m}$ and a core diameter of $12 \mu \mathrm{m}$ and the fibres were singlemode at the wavelength of operation $(1520-1570 \mathrm{~nm})$. The difference in the refractive index between core and cladding was 0.0086 .

\section{A. Etching}

To reduce the cladding thickness, the POF was etched using the solvent etching technique as described in ref [20]. A laboratory grade acetone with $99.5 \%$ concentration was mixed with methanol by 1:1 ratio and then one end of the fibre is inserted into the mixture solution and then removed and cleaned. The etching time depends on the required cladding thickness and in our experiment, to obtain the lowest diameter of $105 \mu \mathrm{m}$, the fibre was immersed in the solution for approximately 6 minutes and the etching rate was around 14 $\mu \mathrm{m} /$ minute. A microscopic image of the fibres before (185 $\mu \mathrm{m})$ and after etching $(105 \mu \mathrm{m})$ are shown in inset of Fig. 1.

To investigate the effect of etching on the material properties of these polymer fibre, Young's modulus and absorption spectrum of the etched polymer fibres are experimentally obtained [21], [22]. The estimated Young's modulus from the stress-strain data obtained from the experiment for fibres with different diameters is shown in Table I. From the table it is clear that Young's modulus of the polymer fibre has significantly reduced with reductions in the fibre diameter through etching. To further verify this result we have used a different sample (sample-2) of PMMA fibres which have different dopant concentrations and proceed with the same experiment for that fibres. Two different diameters of unetched fibre, $450 \mu \mathrm{m}$ and $324 \mu \mathrm{m}$ are tested which resulted the same Young's modulus of $1.05 \mathrm{GPa}$. But when we etched down the $450 \mu \mathrm{m}$ fibre to $340 \mu \mathrm{m}$, Young's modulus is reduced to $0.36 \mathrm{GPa}$. From the table it is clear that Young's modulus of the polymer fibre has significantly reduced with reduction in the fibre diameter through etching.

To further prove the concept, we have used a mathematical equation where the net Young's modulus of the POF is the combination of core and cladding Young's modulus which can be expressed as:

$$
E_{c o m}=\frac{E_{c o} A_{c o}+E_{c l} A_{c l}}{A_{c o}+A_{c l}}
$$

where $E_{c o}$ and $E_{c l}$ are the Young's modulus of core and cladding and $A_{c o}$ and $A_{c l}$ are the surface area of core and cladding. We have estimated the possible change in Young's modulus due to a change in cladding diameter which is shown in Fig. 2. In this case, we used core diameter is $10 \mu \mathrm{m}$ and cladding diameter varies from $50 \mu \mathrm{m}$ to $250 \mu \mathrm{m}$ and also assuming a maximum difference in Young's modulus of $20 \%$ between the core and cladding. From the figure, it is clear that due to smaller size of the core, the impact of cladding diameter change and the small difference in the Young's modulus between core and cladding does not have any significant impact in the net Young's modulus of the POF. The estimated difference in the worst case scenario (20 $\%$ difference between core and cladding Young's modulus) between the $55 \mu \mathrm{m}$ and $245 \mu \mathrm{m}$ cladding diameter is 0.0018 , which is negligible. This confirms that the observed change in 


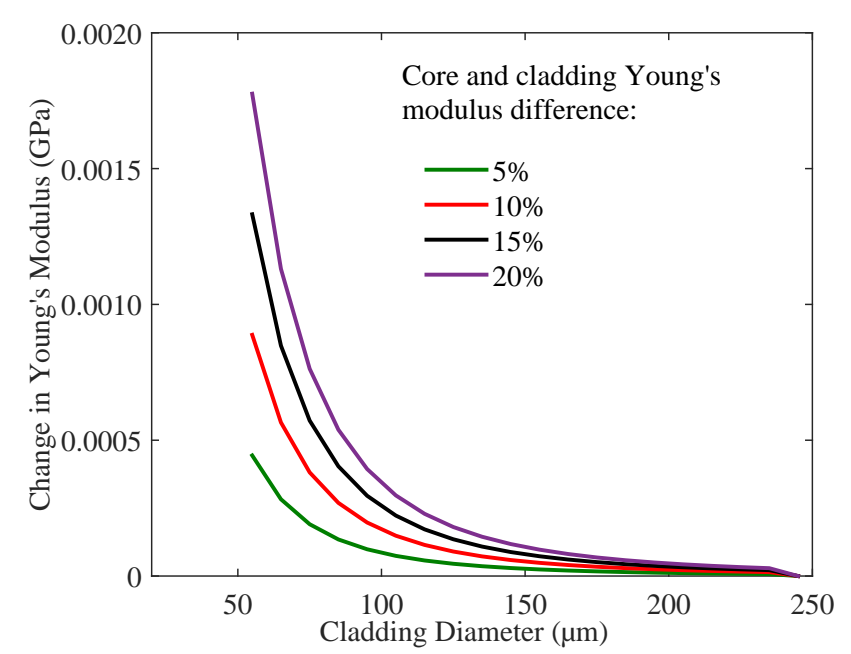

Fig. 2. Change in Young's modulus due to cladding diameter variation

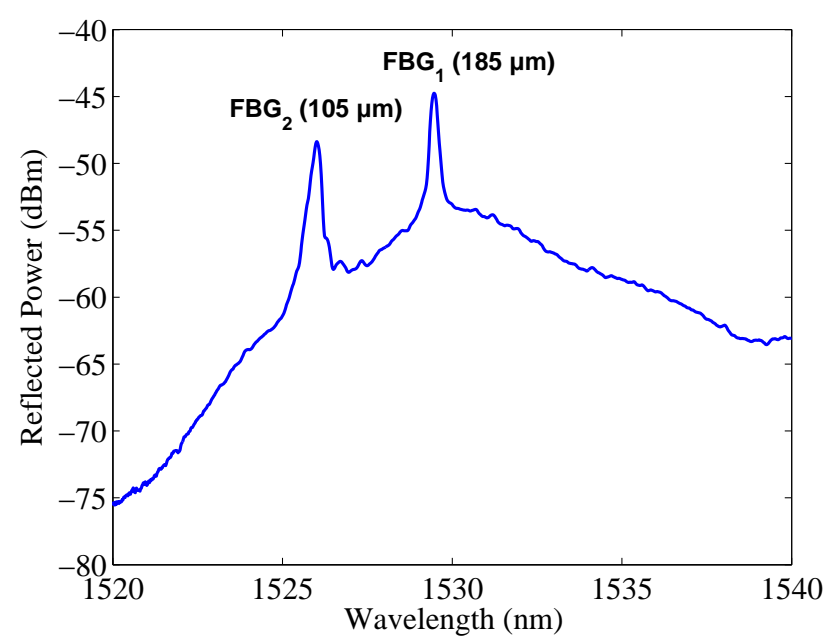

Fig. 3. Reflection spectrum of fabricated FBG pair (185 and $105 \mu \mathrm{m})$

Young's modulus of the POF with different diameter is mainly due to solvent etching. The observed reduction in Young's modulus is due to two reasons; the irreversible relaxation of the polymer chain of the material due to the solvent absorption and also due to the stress relaxation of fiber due to cladding diameter reduction. When the fiber is immersed in the etching solution, the internal stress distribution of the fiber varies with fiber diameter reduction [23] which changes the fiber material properties. So the combined effect of polymer chain relaxation and fiber stress relaxation can soften the fiber material that reduces the Young's modulus.

The modified material properties of an etched polymer fibre can enhance its intrinsic sensing capabilities (strain, temperature and pressure) which is reported in ref [21]. The observed intrinsic changes in strain and temperature sensitivity of etched and un-etched POFBGs lead to the possibility of developing a sensing configuration for simultaneous strain and temperature measurements.

\section{B. Sensor Structure}

To develop a sensor that can measure temperature and strain simultaneously with high sensitivity, a configuration of a POFBG sensor shown in Fig. 1 is used, where an FBG pair with one grating in the etched and another in the un-etched sections. As we already know that etched fibre has different Young's modulus than un-etched fibre, so FBG written on etched fibre will have different responses to temperature and strain than FBG on un-etched fibre. Then strain and temperature change can be measured by utilizing the well-known characterization matrix as shown below:

$$
\left[\begin{array}{c}
\Delta \epsilon \\
\Delta T
\end{array}\right]=\frac{1}{K_{1 \epsilon} K_{2 T}-K_{2 \epsilon} K_{1 T}}\left[\begin{array}{cc}
K_{2 T} & -K_{1 T} \\
-K_{2 \epsilon} & K_{1 \epsilon}
\end{array}\right]\left[\begin{array}{l}
\Delta \lambda_{1} \\
\Delta \lambda_{2}
\end{array}\right]
$$

where $\Delta \lambda_{1}$ and $\Delta \lambda_{2}$ are the net wavelength shift and $K_{1 \varepsilon}, K_{1 T}$ and $K_{2 \varepsilon}, K_{2 T}$ are the strain and temperature sensitivities of the un-etched and etched POFBG respectively. Thus, by exploiting their different strain and temperature sensitivities and using this simple configuration, the strain and temperature can be simultaneously measured with very high sensitivity.

\section{Polymer FBG PAIR}

The Bragg gratings were fabricated by standard phase-mask technique using a $50 \mathrm{~mW}$ Kimmon IK series He-Cd laser emitting light at $325 \mathrm{~nm} \mathrm{[6],} \mathrm{[24].} \mathrm{The} \mathrm{phase} \mathrm{mask} \mathrm{was} 10 \mathrm{~mm}$ long and suitable for a $320 \mathrm{~nm}$ wavelength and can produce $1 \mathrm{~cm}$ long gratings with a peak wavelength of circa $1530 \mathrm{~nm}$ in the single-mode polymer fibre used in this experiment. To observe the Bragg grating reflection or transmission spectrum, a high power broadband source is used which operates in a wavelength range of $1520-1590 \mathrm{~nm}$ with a peak power of circa $1530 \mathrm{~nm}$. In the course of the grating inscription, the reflection spectra of the grating were monitored by an optical spectrum analyser (Agilent OSA 86140B) and the resolution bandwidth is $0.2 \mathrm{~nm}$.

To fabricate the second grating into the same fibre, a method is followed described by Rajan et al [25]. In this technique, a grating is inscribed at a zero strain condition and then an elongation of $0.2 \%$ is applied to the fibre using a translation stage and the position of the UV exposed region is changed by moving the fibre using a motorized translation stage. In a strained condition,the grating period of the fibre after releasing the strain will change by a factor of $\frac{\Lambda \cdot E_{l}}{100}$, where $E_{l}$ is the percentage of elongation applied to the fibre. As a result, the grating period is changed and the FBG exhibits a different peak wavelength. So for an FBG inscribed in an elongated condition, the peak reflected wavelength can be expressed as;

$$
\lambda_{B}=2 n_{e f f} \Lambda\left(1-\frac{E_{l}}{100}\right)
$$

A reflection spectrum of a polymer FBG pair is shown in Fig. 3 where an FBG pair with one grating is in the etched section and another in the un-etched section $(3 \mathrm{~cm}$ apart) and both having distinct peak reflected wavelengths. To use the fabricated POFBGs for simultaneous measurement, POFBG pigtails are fabricated by gluing the POF to a silica fibre pigtail using a UV curable glue and the glued section is protected 


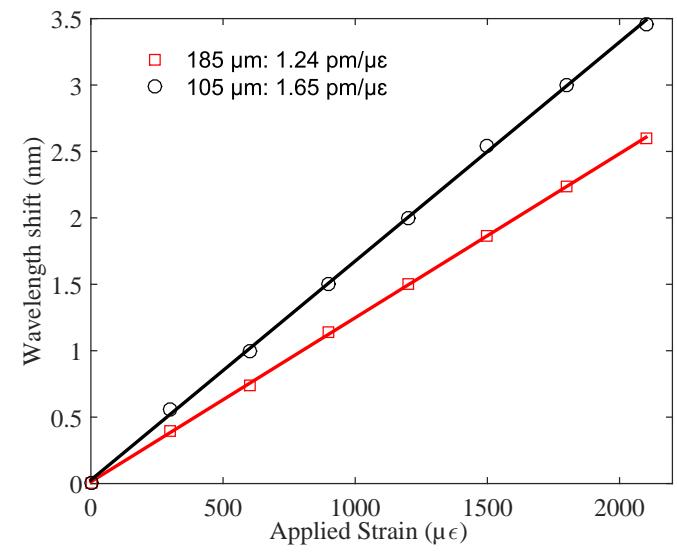

(a)

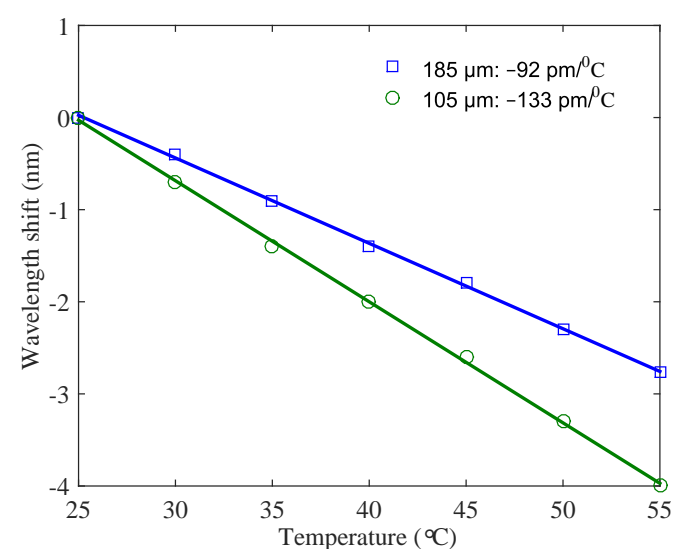

(b)

Fig. 4. (a) Strain response of grating pair (b) Temperature response of grating pair

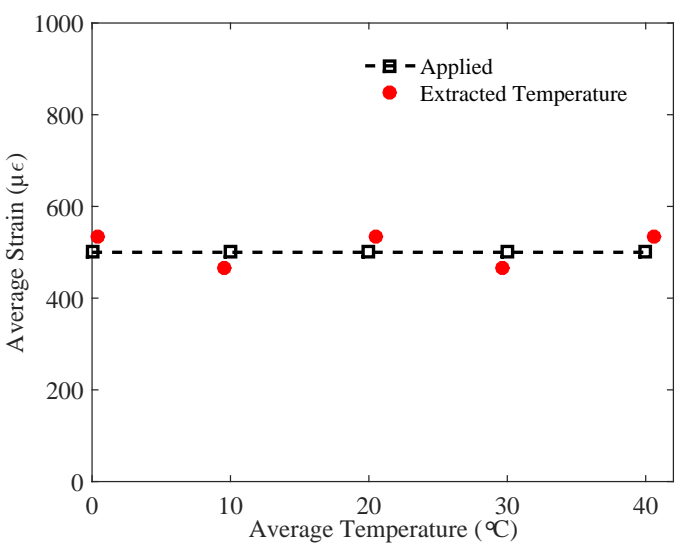

(a)

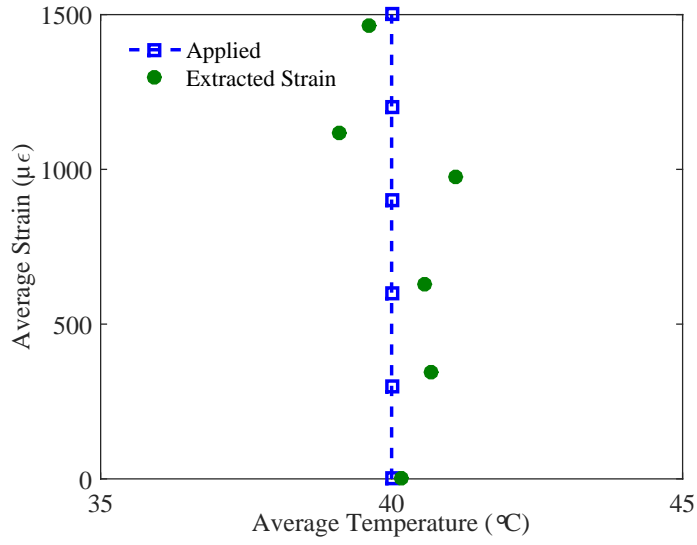

(b)

Fig. 5. (a) Sensor output for applied temperature at constant strain (b) Sensor output for applied strain at constant temperature

using plastic capillary tube. Upon curing this forms a stable POFBG pigtail.

\section{EXPERIMENTAL RESUlts}

\section{A. Strain and Temperature Response of Etched and Un-etched POFBG}

The individual strain and temperature responses of POFBGs fabricated in etched and un-etched fibres are measured. To measure the strain, a standard procedure is followed where a known elongation is applied to the fibre using a translation stage setup. The strain sensitivities of two different diameter polymer FBGs are measured and are shown in Fig. 4(a). Here a positive Bragg wavelength shift is observed for an increase in strain because the dominant effect is the refractive index change than the grating period change. From the figure it can be seen that the Bragg grating inscribed on un-etched fibre exhibits a strain sensitivity of $1.24 \mathrm{pm} / \mu \epsilon$, while a Bragg grating inscribed on etched fibre with diameter of $105 \mu \mathrm{m}$ shows an enhanced sensitivity of $1.65 \mathrm{pm} / \mu \epsilon$. This is due to the lower Young's modulus at etched section than un-etched section of the fibre.
To measure the temperature sensitivities of polymer FBGs, a Peltier cooler based system controlled by a temperature controller is used, which has an accuracy of $\pm 0.1{ }^{\circ} \mathrm{C}$, and the temperature of gratings are varied from $25{ }^{0} \mathrm{C}$ to $55{ }^{\circ} \mathrm{C}$ at $5^{0} \mathrm{C}$ intervals. Due to negative thermo-optic coefficient of the polymer fibre, a negative Bragg wavelength shift is observed for an increase in temperature. The calculated temperature sensitivities from the temperature induced wavelength shifts are shown in Fig. 4(b). The un-etched $185 \mu \mathrm{m}$ polymer FBG exhibits a temperature sensitivity of $-92 \mathrm{pm} /{ }^{\circ} \mathrm{C}$, while a 105 $\mu \mathrm{m}$ etched polymer FBG exhibits a temperature sensitivity of $-133 \mathrm{pm} /{ }^{0} \mathrm{C}$. This difference is expected as a material's Young's modulus is inversely proportional to its thermal expansion coefficient. This means that a decrease in the Young's modulus of the etched fiber increases their thermal expansion coefficient, that have a considerable influence on the thermal sensitivity of a sensors based on etched polymer fibres.

The observed temperature and strain sensitivities for unetched and etched diameter fibre are summarized in Table II where it is clear that etched fibre exhibits higher intrinsic sensitivity than un-etched fibres. 
TABLE II

MEASURED SENSITIVITY PARAMETERS OF THE BRAGG GRATINGS

\begin{tabular}{|c|c|c|}
\hline Fiber Diameter $(\mu \mathbf{m})$ & Temperature Sensitivity $\left(\mathbf{p m} /{ }^{0} \mathbf{C}\right)$ & Strain Sensitivity $(\mathbf{p m} / \mu \epsilon)$ \\
\hline $\mathbf{1 8 5}$ (un-etched) & -92 & 1.24 \\
\hline $\mathbf{1 0 5}$ (etched) & -133 & 1.65 \\
\hline
\end{tabular}

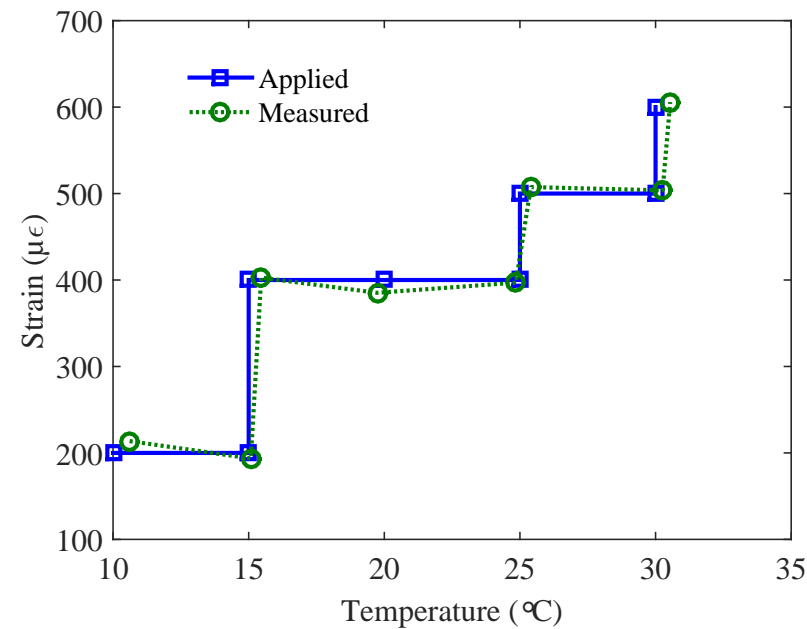

Fig. 6. Sensor responses for different temperatures and strains

\section{B. Simultaneous Measurement}

Since both polymer FBGs exhibited different sensitivities toward the measurands, it is possible to use this sensing head for simultaneous measurements of strain and temperature variations. Due to different diameter section of the sensor it is obvious that both POFBG will have different strain effects. So we consider the average strain applied to the whole fibre and using the measured individual sensitivity coefficients, Eq. 2 can be rewritten as:

$$
\left[\begin{array}{c}
\Delta \epsilon \\
\Delta T
\end{array}\right]=\left[\begin{array}{cc}
10.1346 & -7.0104 \\
0.1257 & -0.0945
\end{array}\right]\left[\begin{array}{c}
\Delta \lambda_{1} \\
\Delta \lambda_{2}
\end{array}\right]
$$

To evaluate the performance of this scheme, the sensor head underwent strain variations upto $1500 \mu \epsilon$ at $300 \mu \epsilon$ step interval at a constant temperature $\left(\mathrm{T}=40^{\circ} \mathrm{C}\right)$ and vice versa, i.e. temperature variations in a range $0-40{ }^{\circ} \mathrm{C}$ at $10{ }^{\circ} \mathrm{C}$ intervals for a specific applied strain $(\epsilon=500 \mu \epsilon)$. Inserting the values of the wavelength shifts exhibited by the 185 $\mu \mathrm{m}$ and $105 \mu \mathrm{m}$ POFBGs and also the individual strain and temperature sensitivities into Eq. 2, strain and temperature changes are calculated. Fig. 5(a) shows the sensor output for temperature changes $0-40{ }^{0} \mathrm{C}$ at a constant strain of 500 $\mu \epsilon$ and Fig. 5(b) shows the sensor result for strain changes $0-1500 \mu \epsilon$ at constant temperature change of $40{ }^{0} \mathrm{C}$. In Fig. 5(a) when strain is constant, then it makes $\pm 0.50{ }^{0} \mathrm{C}$ and $\pm 35.05 \mu \epsilon$ errors for temperature and strain respectively. Similarly when the temperature is constant at Fig. 5(b), then there are $\pm 57.51 \mu \epsilon$ and $\pm 0.77{ }^{\circ} \mathrm{C}$ errors for strain and temperature respectively.

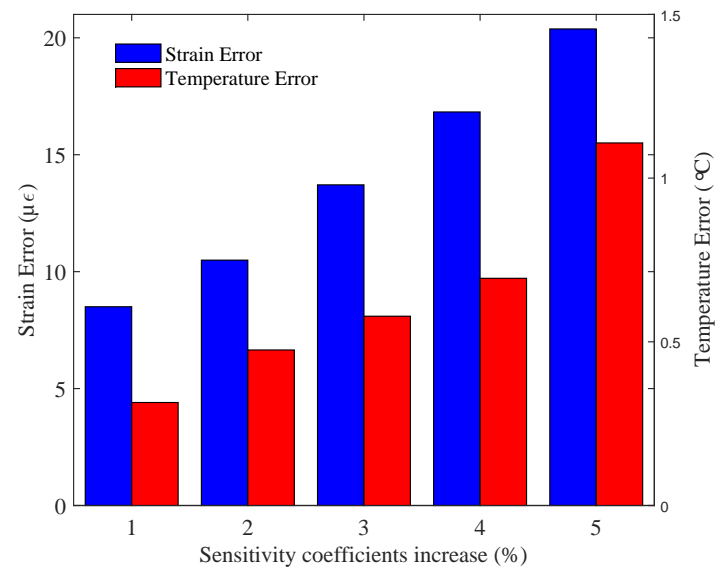

(a)

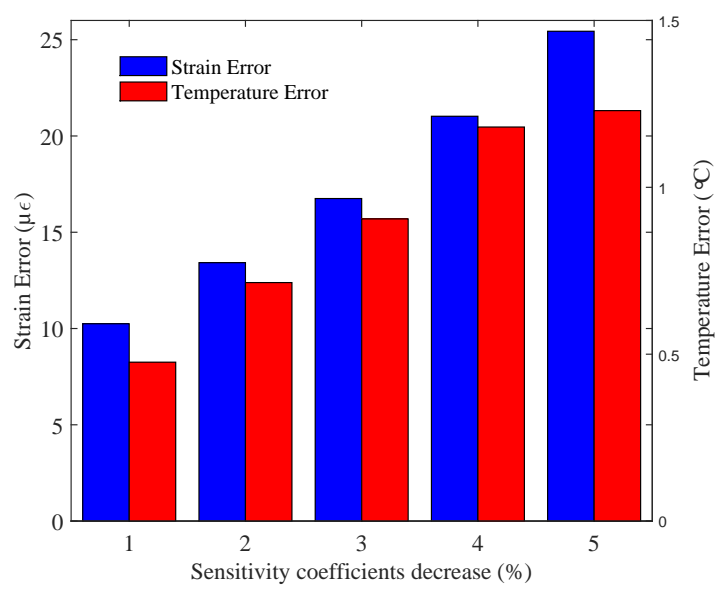

(b)

Fig. 7. Error analysis due to (a) increases in sensitivity coefficients (b) decreases in sensitivity coefficients

To test the capability of the sensor in simultaneous measurements, we experimentally applied both temperature and strain simultaneously and the results are shown in Fig. 6. For example, firstly we applied a $10{ }^{\circ} \mathrm{C}$ temperature and $200 \mu \mathrm{m}$ strain to the sensor and measured their respective wavelength shifts that corresponds to temperature and strain. Secondly, we increased the temperature to $15{ }^{\circ} \mathrm{C}$ at a $200 \mu \mathrm{m}$ strain and then changed the strain to $400 \mu \mathrm{m}$ and measured the respective wavelength shifts in each case. Then using Eq. 4, we can transfer the wavelength shifts to their respective temperature and strain values. In that way, by randomly changing the input temperature and strain, we can simultaneously measure both 
which shows the rms deviations of strain and temperature \pm $8.42 \mu \epsilon$ and $\pm 0.39{ }^{\circ} \mathrm{C}$ respectively. This performance is globally acceptable for many applications that require simultaneous measurements of strain and temperature.

\section{ERror ANALYSIS}

One of the important steps in developing fibre optic strain and temperature sensors is accurately calibrating them, which requires precise determinations of their thermal and strain sensitivities. The change in matrix coefficients due to the different thermal and strain sensitivities cause an errors in the determination of the sensor's output. Fig. 7(a) shows the rms deviations when the individual strain and temperature sensitivity coefficients increase between 1 to $5 \%$. For a 5 $\%$ increase in sensitivity coefficients, it shows $\pm 20.38 \mu \epsilon$ and $\pm 1.11{ }^{\circ} \mathrm{C}$ errors for the strain and temperature measurements respectively. Similarly, we have examined the test when strain and temperature sensitivity coefficient decreases $1-5 \%$ and the result shows in Fig. 7(b). Here for a $5 \%$ decrease in sensitivity coefficients, it shows $\pm 25.44 \mu \epsilon$ and $\pm 1.23{ }^{\circ} \mathrm{C}$ errors for the strain and temperature measurements respectively. From that two graphs it's clear that, if the matrix elements are determined accurately, then the sensor's measurement error rate is relatively low. But if a $1 \%$ error is introduced into the matrix elements, then the error rate for the sensor increases. This strongly indicates that the performance of any simultaneous measurement sensor is greatly influenced by obtaining accurate determinations of the matrix coefficients.

\section{CONCLUSION}

In this paper, we have demonstrated a novel sensing configuration for simultaneous measurement of strain and temperature based on a Bragg grating pair, with one grating in the etched and the other in un-etched region of polymer fibre. This sensor configuration offers a number of advantages over previously reported techniques, including high intrinsic sensitivity, low cost and ease in fabrication. Hence it can be used in any applications where simultaneous measurements are required. A maximum errors of $\pm 8.42 \mu \epsilon$ and $\pm 0.39{ }^{\circ} \mathrm{C}$ are observed in our experimental results. The accuracy of the matrix coefficients due to change in the thermal and strain sensitivities of the sensor is also investigated. Our analysis of measurement accuracy showed that, it requires accurate measurements of individual sensitivity values to ensure the acceptable accuracy of temperature and strain values recovery, otherwise the error in matrix coefficients greatly degrade the sensor's accuracy.

\section{ACKNOWLEDGMENT}

We also thank for the support by Open Funds of IPOC (BUPT, IPOC2014B010), SKLFPM (DHU, LK1502), and State Key Laboratory of Advanced Optical Communication Systems Networks, China.

\section{REFERENCES}

[1] K. Peters, "Polymer optical fiber sensorsa review," Smart Materials and Structures, vol. 20, no. 1, pp. 013002 (1-17), 2011.

[2] G. D. Peng, Z. Xiong, and P. L. Chu, "Photosensitivity and gratings in dye-doped polymer optical fibers," Optical Fiber Technology, vol. 5, no. 2, pp. 242-251, April 1999.
[3] K. Kalli, H. L. Dobb, D. J. Webb, K. Carroll, C. Themistos, M. Komodromos, and et al., "Development of an electrically tuneable bragg grating filter in polymer optical fibre operating at $1.55 \mu \mathrm{m}$," Measurement Science and Technology, vol. 18, pp. 3155-3164, 2007.

[4] D. J. Webb, K. Kalli, C. Zhang, I. P. Johnson, X. F. Chen, D. S Rodriguez, and et al., "Applications of polymer fiber grating sensors," in 18th Int. Conf. Plastic Opt. Fibers, 2009.

[5] W. Zhang, D. J. Webb, and G. D. Peng, "Investigation into time response of polymer fiber bragg grating based humidity sensors," Journal of Lightwave Technology, vol. 30, no. 8, pp. 1090-1096, April 2012.

[6] K. Bhowmik, G.-D. Peng, Y. Luo, E. Ambikairajah, V. Lovric, W. Walsh, and G. Rajan, "Experimental study and analysis of hydrostatic pressure sensitivity of polymer fibre bragg gratings," Lightwave Technology, Journal of, vol. 33, no. 12, pp. 2456-2462, June 2015.

[7] M. G. Xu, J. L. Archambault, L. Reekie, and J. P. Dakin, "Discrimination between strain and temperature effects using dual-wavelength fibre grating sensors," Electronics Letters, vol. 30, no. 13, pp. 1085-1087, Jun 1994.

[8] S. W. James, M. L. Dockney, and R. P. Tatam, "Simultaneous independent temperature and strain measurement using in-fibre bragg grating sensors," Electronics Letters, vol. 32, no. 12, pp. 1133-1134, Jun 1996.

[9] H. B. Liu, H. Y. Liu, G. D. Peng, and P. L. Chu, "Strain and temperature sensor using a combination of polymer and silica fibre bragg gratings," Optics Communications, vol. 219, no. 16, pp. $139-142,2003$.

[10] X. Shu, Y. Liu, D. Zhao, B. Gwandu, F. Floreani, L. Zhang, and I. Bennion, "Dependence of temperature and strain coefficients on fiber grating type and its application to simultaneous temperature and strain measurement," Opt. Lett., vol. 27, no. 9, pp. 701-703, May 2002.

[11] O. Frazo, M. J. N. Lima, and J. L. Santos, "Simultaneous measurement of strain and temperature using type i and type iia fibre bragg gratings," Journal of Optics A: Pure and Applied Optics, vol. 5, no. 3, p. 183, 2003.

[12] P. Cavaleiro, F. Araujo, L. Ferreira, J. Santos, and F. Farahi, "Simultaneous measurement of strain and temperature using bragg gratings written in germanosilicate and boron-codoped germanosilicate fibers," Photonics Technology Letters, IEEE, vol. 11, no. 12, pp. 1635-1637, Dec 1999.

[13] H. J. Patrick, G. M. Williams, A. D. Kersey, J. R. Pedrazzani, and A. M. Vengsarkar, "Hybrid fiber bragg grating/long period fiber grating sensor for strain/temperature discrimination," Photonics Technology Letters, IEEE, vol. 8, no. 9, pp. 1223-1225, Sept 1996.

[14] J. Jung, H. Nam, J. H. Lee, N. Park, and B. Lee, "Simultaneous measurement of strain and temperature by use of a single-fiber bragg grating and an erbium-doped fiber amplifier," Appl. Opt., vol. 38, no. 13, pp. 2749-2751, May 1999.

[15] L. Xiaohong, W. Dexiang, Z. Fujun, and D. Enguang, "Simultaneous independent temperature and strain measurement using one fiber bragg grating based on the etching technique," Microwave and Optical Technology Letters, vol. 43, no. 6, pp. 478-481, 2004.

[16] M. Song, B. Lee, S. B. Lee, and S. S. Choi, "Interferometric temperatureinsensitive strain measurement with different-diameter fiber bragg gratings," Opt. Lett., vol. 22, no. 11, pp. 790-792, Jun 1997.

[17] Y. Dong, L. Chen, and X. Bao, "High-spatial-resolution time-domain simultaneous strain and temperature sensor using brillouin scattering and birefringence in a polarization-maintaining fiber," Photonics Technology Letters, IEEE, vol. 22, no. 18, pp. 1364-1366, Sept 2010.

[18] M. Song, S. B. Lee, S. S. Choi, and B. Lee, "Simultaneous measurement of temperature and strain using two fiber bragg gratings embedded in a glass tube," Optical Fiber Technology, vol. 3, no. 2, pp. 194-196, April 1997.

[19] G. D. Peng, P. L. Chu, X. Lou, , and R. A. Chaplin, "Fabrication and characterization of polymer optical fibers," Journal of Electrical and Electronics Engineering, vol. 15, pp. 289-296, 1995.

[20] D. F. Merchant, P. J. Scully, and N. F. Schmitt, "Chemical tapering of polymer optical fibre," Sensors and Actuators A: Physical, vol. 76, no. 13, pp. 365 - 371, August 1999.

[21] K. Bhowmik, G.-D. Peng, E. Ambikairajah, V. Lovric, W. Walsh, B. Prusty, and G. Rajan, "Intrinsic high-sensitivity sensors based on etched single-mode polymer optical fibers," Photonics Technology Letters, IEEE, vol. 27, no. 6, pp. 604-607, March 2015.

[22] "Standard test method for tensile strength and young's modulus of fibers," Active Standard ASTM C1557, vol. 15.01, August 2003.

[23] K.-S. Lim, H.-Z. Yang, W.-Y. Chong, Y.-K. Cheong, C.-H. Lim, N. M. Ali, and H. Ahmad, "Axial contraction in etched optical fiber due to internal stress reduction," Opt. Express, vol. 21, no. 3, pp. 2551-2562, Feb 2013. 
[24] G. Rajan, M. Ramakrishnan, Y. Semenova, E. Ambikairajah, G. Farrell, and G. D. Peng, "Experimental study and analysis of a polymer fiber bragg grating embedded in a composite material," Journal of Lightwave Technology, vol. 32, no. 9, pp. 1726-1733, May 2014.

[25] G. Rajan, M. Noor, E. Ambikairajah, and G.-D. Peng, "Inscription of multiple bragg gratings in a single-mode polymer optical fiber using a single phase mask and its analysis," Sensors Journal, IEEE, vol. 14, no. 7, pp. 2384-2388, July 2014.

Kishore Bhowmik is a PhD student in the School of Electrical Engineering and Telecommunications at the University of New South Wales (UNSW), Sydney, Australia. He received the B.Sc. degree in Electronics and Telecommunication Engineering from the Rajshahi University of Engineering \& Technology (RUET), Bangladesh in 2010.

His current research interests include polymer optical fibres, polymer fibre Bragg gratings, optical fibre sensors and its applications in biomedical engineering. He is a student member of the IEEE Sensors Council and the SPIE.

Gang-Ding Peng received the B.Sc. degree in physics from Fudan University, Shanghai, China, in 1982, the M.Sc. degree in applied physics, and the Ph.D. degree in electronic engineering from Shanghai Jiao Tong University, Shanghai, in 1984 and 1987, respectively.

He was a Lecturer with Jiao Tong University, Shanghai, from 1987 to 1988. He was a Post-Doctoral Research Fellow with the Optical Sciences Centre, Australian National University, Canberra, Australia, from 1988 to 1991. He has been with the UNSW, Australia, since 1991, was a Queen Elizabeth II Fellow from 1992 to 1996, and is currently a Professor with the same university. His current research interests include optical fiber and waveguide devices, silica and polymer optical fibers, optical fiber sensors, and nonlinear optics.

Prof. Peng is a fellow and life member of both the Optical Society of America and the SPIE.

Yanhua Luo received the Bachelors degree in polymer materials and engineering and the Ph.D. degree in chemistry from the University of Science and Technology of China, Hefei, China, in 2004 and 2009, respectively.

He has visited the School of Electrical Engineering and Telecommunications, UNSW, Australia, as a Visiting Student twice to do research about polymer optical fibre gratings. He is currently a Lab Manager of Photonic and Optical Communications Laboratory, UNSW. He has published over 44 journal papers and 17 conference papers. His current research interests include photonic materials, including sensitization luminescence of rare earth complexes, polymer fibre Bragg gratings, and specialty silica optical fiber.

Eliathamby Ambikairajah (M'91) received the B.Sc. (Eng.) degree from the University of Sri Lanka, and the Ph.D. degree in signal processing from Keele University, U.K.

He was appointed as the Head of Electronic Engineering and later Dean of Engineering with the Athlone Institute of Technology, Ireland, from 1982 to 1999 . He is currently the Head of the School of Electrical Engineering and Telecommunications, UNSW, Australia. He has authored or co-authored more than 250 conference and journal papers, and is a regular reviewer for several IEEE, IET and other journals and conferences. His current research interests include speech enhancement, speaker recognition, language identification, emotion detection, and biomedical signal processing.

Prof. Ambikairajah received the Vice-Chancellors Award for Teaching Excellence in 2004 for his innovative use of educational technology, the School Awards for Teaching Excellence in 2003. He is currently a fellow and a Chartered Engineer of the IET, U.K., and the Engineers Australia.

Vedran Lovric received the B.Eng degree from the University of Technology (UTS), Australia, and the M.Eng.BME degree in Biomedical Engineering from University of New South Wales (UNSW), Australia in 2005 and 2007 respectively and the Ph.D. degree from the UNSW, Australia, in 2012.

He is currently a research fellow at the Surgical and Orthopaedic Research Laboratory with the Prince of Wales Clinical School, UNSW, Australia. He is also the scientific arm of the Australian Biotechnologies Medical Advisory Council. His current research interests include design of biomaterial for orthopaedic applications, Supercritical $\mathrm{CO}_{2}$ sterilization techniques, biomechanics of biomaterial, and tendon-bone healing.

W. R. Walsh received his undergraduate degrees in Chemistry and Biology (Bucknell University, Lewisburg PA) followed by a PhD in Biomedical Engineering from Rutgers University and Robert Wood Johns Medical School in New Jersey. Prof. Walsh is the Director of the Surgical and Orthopaedic Research Laboratories in the Prince of Wales Clinical School, UNSW, Australia.

His research is at the interface between implanted materials and the connective tissues of the body as it relates to orthopaedic, plastic and reconstructive and vascular surgery. This includes both autograft, allograft and synthetic biomaterials and understanding and improving these materials in areas of clinical need.

Ginu Rajan received the B.Sc. degree in physics from the University of Kerala, Kerala, India, and the M.Sc. degree in applied physics from Mahatma Gandhi University, Kerala, in 2000 and 2002, respectively, and the Ph.D. degree from the Dublin Institute of Technology (DIT), Dublin, Ireland, in 2008.

He was a Research Trainee with the Indian Institute of Astrophysics, from 2003 to 2005. From 2009 to 2012, he was a Project Manager and Research Associate with the Photonics Research Centre, DIT, in collaboration with the Warsaw University of Technology, Warsaw, Poland. He was an Assistant Lecturer at DIT and Vice-Chancellors Research Fellow with UNSW, Australia. He is currently a Vice-Chancellors Research Fellow with The University of Wollongong, Australia. He has published over 70 articles in journals, conferences, and book chapters. His current research interests include optical fiber sensors and its applications in biomedical engineering, FBG interrogation systems, photonic crystal fiber sensors, polymer fiber sensors, smart structures, and physics of photonic devices. 